\title{
Human recombinant erythropoietin (rEpo) has no effect on tumour growth or angiogenesis
}

\author{
ME Hardee', JP Kirkpatrick ${ }^{2}$, S Shan ${ }^{2}$, SA Snyder ${ }^{2}$, Z Vujaskovic ${ }^{2}$, ZN Rabbani ${ }^{2}$, MW Dewhirst ${ }^{2}$ and \\ KL Blackwell*,2,3
}

'Department of Pathology, Duke University Medical Center, Box 3893, Durham, NC 2771 0, USA; 'Department of Radiation Oncology, Duke University Medical Center, Box 3893, Durham, NC 27710, USA; ${ }^{3}$ Department of Hematology-Oncology, Duke University Medical Center, Box 3893 , Durham, NC 27710, USA

\begin{abstract}
Tumour hypoxia has been shown to increase mutation rate, angiogenesis, and metastatic potential, and decrease response to conventional therapeutics. Improved tumour oxygenation should translate into increased treatment response. Exogenous recombinant erythropoietin ( $r E p o$ ) has been recently shown to increase tumour oxygenation in a mammary carcinoma model. The mechanism of this action is not yet understood completely. The presence of Epo and its receptor (EpoR) have been demonstrated on several normal and neoplastic tissues, including blood vessels and various solid tumours. In addition, rEpo has been shown in two recent prospective, randomized clinical trials to negatively impact treatment outcome. In this study, we attempt to characterize the direct effects of rEpo on tumour growth and angiogenesis in two separate rodent carcinomas. The effect of rEpo on R3230 rat mammary adenocarcinomas, CT-26 mouse colon carcinomas, HCT-I I 6 human colon carcinomas, and FaDu human head and neck tumours, all of which express EpoR, was examined. There were no differences in tumour growth or proliferation (measured by Ki-67) between placebo-treated and rEpo-treated tumours. In the mammary window chamber, vascular length density (VLD) measurements in serial images of both placebo-treated and Epo-treated rats revealed no difference in angiogenesis between the Epo-treated tumours and placebo-treated tumours at any time point. These experiments are important because they suggest that the recent clinical detriment seen with the use of Epo is not due to its tumour growth effects or angiogenesis. These studies also suggest that further preclinical studies need to examine rEpo's direct tumour effects in efforts to improve the therapeutic benefits of Epo in solid tumour patients.

British Journal of Cancer (2005) 93, I350- |355. doi:I0.1038/sj.bjc.6602846 www.bjcancer.com
\end{abstract}

Published online 15 November 2005

(C) 2005 Cancer Research UK

Keywords: erythropoietin; angiogenesis; tumour growth

Low oxygen tension $\left(\mathrm{pO}_{2}\right)$ promotes the selection of cells with reduced apoptotic potential (Graeber et al, 1996), increases the frequency of mutation (Reynolds et al, 1996), induces genes promoting angiogenesis (Dachs and Chaplin, 1998) and decreases radiosensitivity (Bedford and Mitchell, 1974). Clinically, tumour hypoxia is associated with poorer outcome. A variety of approaches have been used to reduce tumour hypoxia with the goal of improving the therapeutic response to chemotherapy and/ or radiation therapy. A recent promising approach in preclinical models involves the administration of exogenous recombinant erythropoietin (rEpo) (Blackwell et al, 2003). Erythropoietin is a glycoprotein growth factor that is essential for erythroid precursors to mature into red blood cells by stimulating growth and preventing apoptosis (Krantz, 1991). It is a clinically important member of the growth factor family, in that it is produced endogenously in the human body and it may be given exogenously

*Correspondence: Assistant Professor KL Blackwell, Departments of Medicine and Radiation Oncology, Duke University Medical Center, Box 3893, Durham, NC 277I0, USA; E-mail: black034@mc.duke.edu Received 13 July 2005; revised 30 August 2005; accepted 2 September 2005; published online 15 November 2005 in the human recombinant form to treat the anemia associated with several chronic diseases. However, there have been several reports suggesting that both Epo and EpoR can be expressed in various neoplastic tissues, raising the possibility of autocrine pathways. A randomized, placebo-controlled trial of rEpo in nonanemic metastatic breast cancer patients was terminated early because of an unexpectedly higher mortality rate in the group receiving rEpo (Leyland-Jones, 2003). Also, a recent trial involving patients with head and neck cancer found that rEpo did not improve the efficacy of standard therapy (Henke et al, 2003). Although the initial results from these two trials are disappointing, their design fails to define a biologic mechanism for why rEpo might lessen treatment effects. This preclinical study examines rEpo's effect on tumour growth and proliferation in two rodent tumour lines and two human tumour lines, as well as its effects on tumour angiogenesis.

\section{MATERIALS AND METHODS}

\section{Animals}

Female BALB/C mice (National Cancer Institute, Washington, DC, USA) were used in the CT-26, HCT-116, and FaDu flank tumour 
studies. Female Fischer 344 rats (Charles River Laboratories, Raleigh, NC, USA) were used for the R3230 flank tumour studies and the mammary window chamber experiments. Tumour cells were injected into the subcutis of the right quadriceps muscle for flank tumours. Following injection of tumour cells or mammary window surgery, the animals were allowed free access to food and water ad libitum. All procedures and experiments were approved by the Duke Institutional Animal Care and Use Committee.

\section{Cell culture}

R3230 (rat mammary adenocarcinoma) and GFP-R3230 (R3230 tumour cells constituitively transfected with green fluorescence protein; as described in (Huang et al, 1999)) tumour cells were maintained in DMEM (Sigma, St Louis, MO, USA) medium. CT-26 (murine colon adenocarcinoma) tumour cells were maintained in RPMI (Sigma, St Louis, MO, USA). HCT-116 (human colon carcinoma) tumour cells were maintained in McCoy's 5A medium (Sigma). FaDu (human head and neck carcinoma) were maintained in MEM supplemented with $1 \%$ sodium pyruvate and $1 \%$ amino-acid solution. All media was supplemented with $10 \%$ FBS (Sigma) and 1\% penicillin/streptomycin (Sigma).

\section{rEpo administration and hematocrit measurements}

Animals in all studies were administered rEpo (Procrit ${ }^{\mathrm{TM}}$, Ortho-

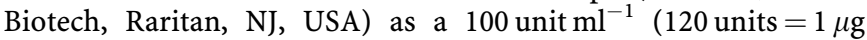
$\mathrm{EPO}$ ) solution in $0.9 \% \mathrm{NaCl}$ via an injection into the subcutis of the nape of the neck at a dose of 2000 units kg $^{-1}$ thrice per week. This dosing is similar to a rEpo dose of $60000-100000 \mathrm{IU}_{\text {dose }}^{-1}$ in humans, as referenced in previous work (Blackwell et al, 2003). Hematocrit was determined in all animals by collecting $50 \mu \mathrm{l}$ of blood in a capillary tube from a tail vein of anesthesized animals, then centrifuging for $1 \mathrm{~min}$ and calculating hematocrit from the ratio of the length of packed RBC's to that of plasma.

\section{Tumour volume}

In rats, tumours were measured transversely and the volume calculated using the formula:

$$
\begin{aligned}
\text { Volume }= & \text { greatest transverse dimension } \\
& \times(\text { orthogonal transverse dimension })^{2} \times \pi / 6
\end{aligned}
$$

where all dimensions are in $\mathrm{mm}$. In mice, tumour volume was determined by the formula:

$$
\text { Volume }=\text { length } \times \text { width } \times \text { height } \times \pi / 6
$$

where all linear dimensions are in $\mathrm{mm}$.

\section{Epo receptor and Ki-67 immunohistochemistry}

Standard immunohistochemical techniques were used to stain for both EpoR and Ki-67. Briefly, IHC was performed using $10 \mu \mathrm{m}$ serial sections of frozen tissue. Sections were fixed in $4^{\circ} \mathrm{C}$ cold acetone for $10 \mathrm{~min}$. After endogenous peroxidase activity was quenched with $3 \%$ hydrogen peroxide for $15 \mathrm{~min}$, tumour sections were blocked with $10 \%$ donkey serum for $15 \mathrm{~min}$. Sections were incubated in primary antibodies (anti-EpoR (M-20), 1:200 (Santa Cruz Biotechnology, Santa Cruz CA, USA); anti-Ki-67, 1:1000 (Novacastra, UK)) overnight at $4{ }^{\circ} \mathrm{C}$. The location of the antibody reactions was visualized with $3,3^{\prime}$-diaminobenzidine tetrahydrochloride (Sigma). For Ki-67 staining, four representative fields at $\times 40$ objective taken from one tumour in each of six animals in both the control and Epo-treatment group were measured. Proliferation index was calculated by manually counting the ratio of Ki-67-positive nuclei to total number of nuclei in each field, and the average proliferation index for each tumour was calculated.

\section{Mammary window chamber assay}

The method used is the same as that described by Shan et al (2003). The assay is an orthotopic variation of the dorsal skin fold window chamber, which is the gold standard for serially measuring in vivo angiogenesis. The epidermis around either the right third or fourth nipple was removed, leaving an intact nipple and surrounding subcutaneous tissue. The nipple was then removed at its base, exposing the nipple sinus. A $1 \mathrm{~mm}^{3}$ piece of Gelfoam (Pharmacia \& Upjohn, Kalamazoo, MI, USA) soaked in $3 \mu \mathrm{l}$ of an R3230-GFP cell suspension of concentration $2 \times 10^{6} \mathrm{cells} \mathrm{ml}^{-1}$ (total of 6000 tumour cells) was inserted into the nipple sinus. This was then covered with an acrylic window, which was sutured in place.

\section{Intravital microscopy}

The mammary windows were imaged serially using a Carl Zeiss MPS intravital microscope (Carl Zeiss, Hanover, MD, USA) every other day, starting on postoperative day 2 and ending on postoperative day 14. The microscope is equipped with a color video camera (Carl Zeiss ZVS-3C75DE) connected to a PC computer with a frame grabber and Scion Image software (Scion Corporation, Frederick, MD). Fluorescence epi-illumination was provided with a $100 \mathrm{~W}$ mercury-arc lamp (AttoArc HBO, Carl Zeiss Inc, USA) and a FITC filter (excitation 450-490 nm and emission $520 \mathrm{~nm}$ ). Images were obtained at low magnification (objective $\times 5)$ and analyzed using Scion Image software. Vascular length density (VLD) was calculated for each image by an observer unaware of the treatment group.

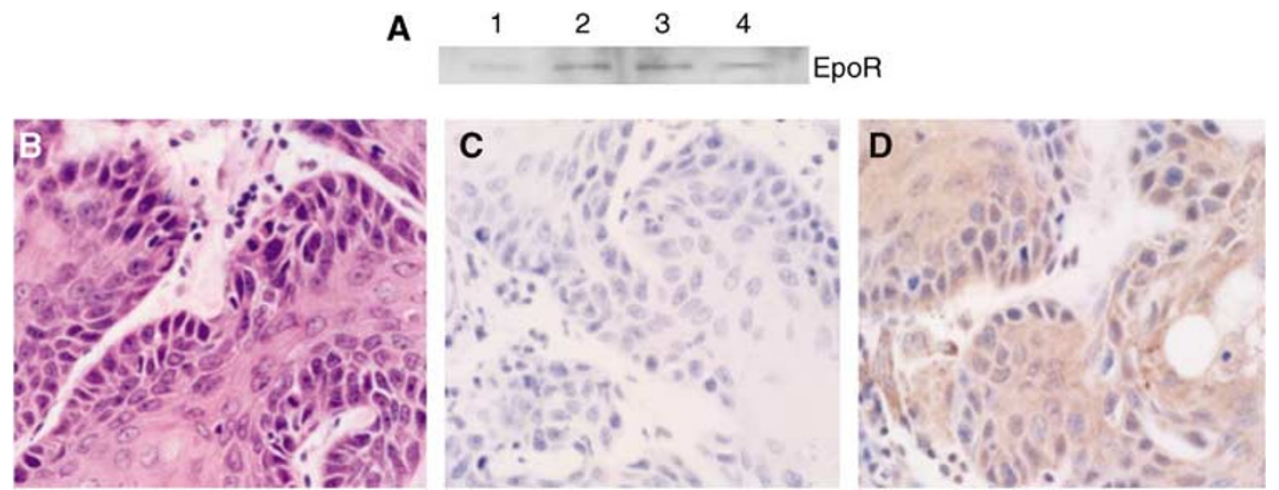

Figure I EpoR status of tumour cell lines. Shown in $(\mathbf{A})$ is an EpoR Western blot on all tumour cell lines used in this study. Positive staining is seen in all the tumour cell lines. Also shown are H\&E (B), negative lgG control $(\mathbf{C})$, and EpoR $(\mathbf{D})$ staining of a representative R3230 flank tumour section taken with a $\times 40$ objective. 


\section{Statistics}

The data on hematocrit, tumour volumes, and VLD were analyzed and treatment groups compared using $t$-test for independent means. In all analyses, two-sided $P$-values less than 0.05 was considered significant.
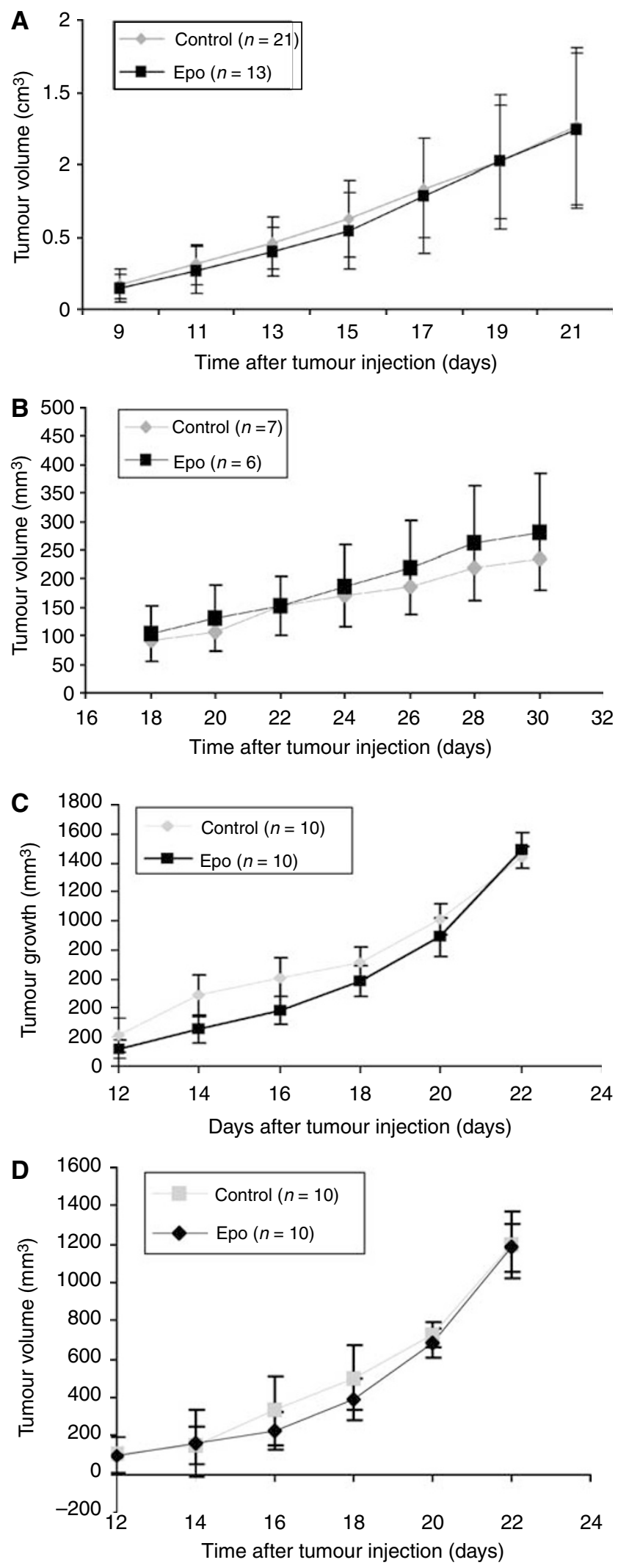

\section{RESULTS}

\section{R3230, CT-26, HCT-116, and FaDu tumours express Epo receptor}

To establish relevance for this study, we determined the EpoR status of each of the four tumour cell lines used. All four tumour cell lines used in this experiment expressed Epo receptor by Western blot (Figure 1A). This was confirmed by immunohistochemical staining for EpoR. Interestingly, the strongest staining for the receptor was in perinecrotic regions (Figure $1 \mathrm{~B}$ ).

\section{Epo increases hematocrit}

In the tumour growth studies, baseline hematocrit of mice was $46.5 \pm 1.5 \%$. Treatment with Epo produced a hematocrit of $60.9 \pm 3.1$ vs $46.7 \pm 2.1 \%$ in the control group after 14 days $(P<0.05)$. In the mammary window chamber experiments, EPO significantly increased the baseline hematocrit $(45.8 \pm 2.6)$ of treated rats by $36 \%$ after 8 days and $40 \%$ at 14 days compared to placebo-treated rats $\left(P=3.2 \times 10^{-8}\right.$ and $4.3 \times 10^{-9}$ at days 8 and 14 , respectively).

\section{Epo does not promote tumour growth or proliferation rate}

Tumour growth was observed in all rats transplanted with R3230 tumour cells and mice transplanted with CT-26, HCT-116, and $\mathrm{FaDu}$ tumour cells. Tumour volumes were similar between the EPO-treated and control groups in each of the four tumour cell lines throughout the length of the study (Figure 2A-D). As measured by Ki-67 immunohistochemical staining, there was no statistical difference between the mean proliferation indices between the EPO-treated and control groups (Figure 3) in any of the tumour cell lines.

\section{Epo does not stimulate tumour angiogenesis}

There was no statistical difference in VLD between the EPO-treated and control groups (Figure 4) at any timepoint $(P>0.05$ at all timepoints). All 20 rats tolerated the surgery well and were healthy immediately postoperatively. At postoperative day 14, 14 of 20 rats retained their mammary windows and had tumours that could be visualized. This represents a success rate of $70 \%$, consistent with that of Shan (Shan et al, 2003). Six animals were unevaluable due to: (1) rats that pulled off their windows $(n=2),(2)$ lack of tumour growth $(n=3)$, or (3) infection/inflammation that prevented tumour visualization $(n=1)$.

\section{DISCUSSION}

Recombinant Epo has recently been shown to improve tumour oxygenation; its mechanism has not been determined. Tumour oxygenation is dependent on a delicate balance between oxygen delivery and oxygen consumption. Previous preclinical work in this area, all done in vitro, has implicated rEpo as promoting tumour growth (Westenfelder and Baranowski, 2000; Yasuda et al,

Figure 2 Volume of tumours grown in BALB/C mice and Fischer 344 rats. CT-26 flank tumours $(\mathbf{A})$ were grown in the right flank of female BALB/C mice, R3230Ac flank tumours (B) were grown in the right flank of female Fischer 344 rats, and FaDu (C) and HCT-II6 (D) flank tumours were grown in the right flank of female athymic mice. rEpo was administered thrice weekly at dose of 2000 units $\mathrm{kg}^{-1}$ for a total of six doses of rEpo after tumours reached $100-200 \mathrm{~mm}^{3}$. Control-treated and rEpo-treated tumour volumes were similar for all tumour types. Shown are mean values \pm standard error of the mean (s.e.m.). T-test for independent samples with two-tailed $P$-value. 

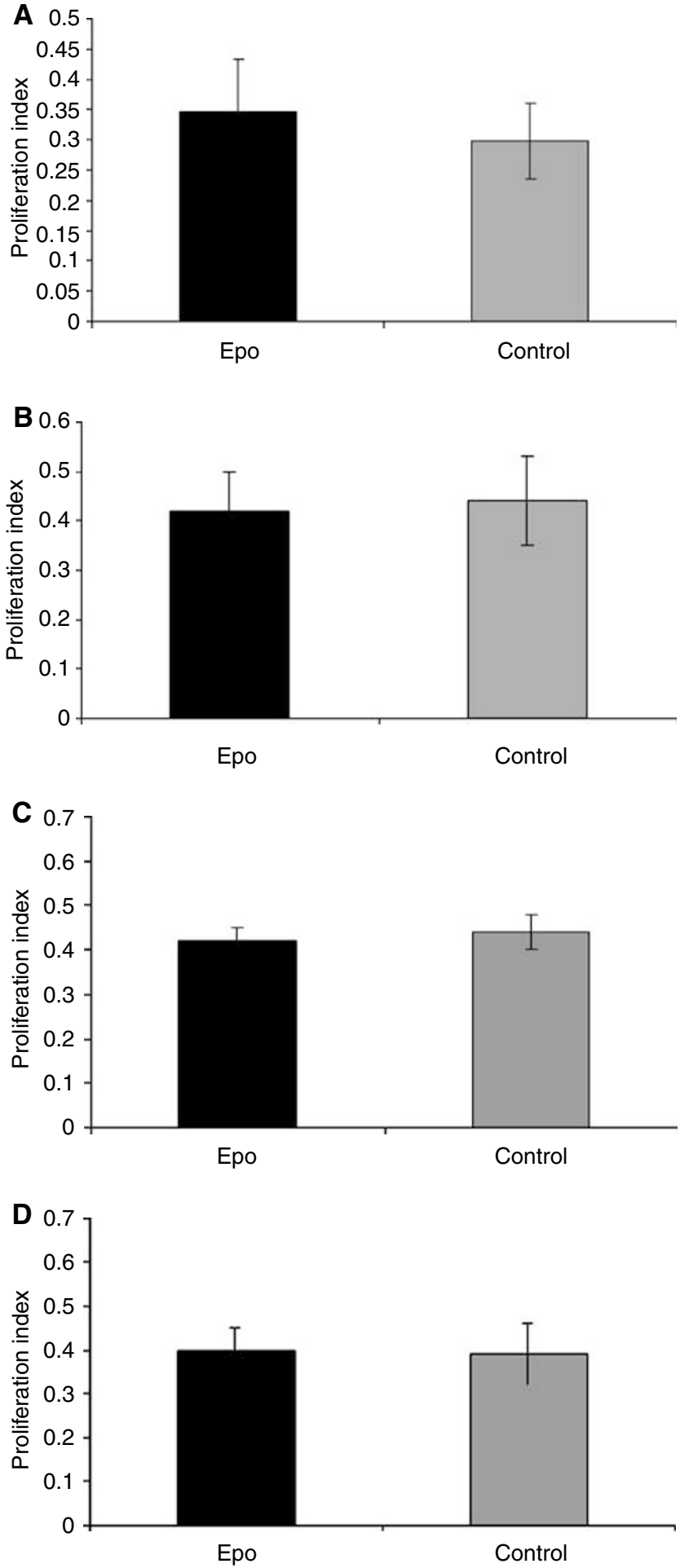

Figure 3 Effect of rEpo on proliferation rate of tumour cells. Sections were taken from (A) CT-26, (B) R3230, (C) FaDu, and (D) HCT-II6 tumours and stained for Ki-67. There was no statistical difference between the mean proliferation index of the rEpo-treated group and that of the control-treated group in any tumour model $(P=0.14,0.23,0.09$, and $0.1 \mathrm{I}$, respectively).

2002) and/or being proangiogenic (Anagnostou et al, 1990; Carlini et al, 1995; Ribatti et al, 1999; Acs et al, 2001; Yasuda et al, 2002). However, no studies have looked at the in vivo effects of therapeutic levels of rEpo on these two processes.
Several studies have suggested that Epo might stimulate angiogenesis by acting to promote proliferation and/or migration of endothelial cells (Anagnostou et al, 1990; Ribatti et al, 1999), inhibiting apoptosis of endothelial cells (Carlini et al, 1999), or by increasing MMP-2 secretion (Ribatti et al, 1999). rEpo has also been shown to increase the number of circulating endothelial cells and endothelial cell progenitors in preclinical models (Monestiroli et al, 2001) and human breast cancer and lymphoma models (Mancuso et al, 2001). Our studies demonstrate the importance of in vivo modeling of rEpo's effects, as we found no effects of rEpo on tumour cell proliferation, tumour growth or angiogenesis.

Previous rEpo study results may be limited in their application by the fact that they examined the effects of rEpo on one particular cell line. In addition, previous studies used supratherapeutic concentrations of recombinant Epo $\left(5-100 \mathrm{U} \mathrm{ml}^{-1}\right.$; Carlini et al, 1995), as compared to levels of Epo achieved clinically to correct hematocrit (1.7-3.6 $\mathrm{U} \mathrm{ml}^{-1}$; (Carlini et al, 1995)). Also, the expression of Epo receptor on the tumour or endothelial cell line that was used was not determined and/or the rEpo was administered over a short period of time.

Our study is strengthened by the fact that we used four separate tumour lines that express the Epo receptor and treated the animals over an extended period. While the aim of this study was to determine the effects of exogenous rEpo on two biologic processes that directly affect tumour oxygenation (tumour growth and angiogenesis), intra-tumoural production of Epo might also influence tumour behavior in an autocrine/paracrine fashion. Evidence for a functional Epo-EpoR system has been reported by several groups: intratumoural and intraperitoneal injections of solube EpoR or neutralizing monoclonal antibody to EpoR resulted in tumour growth delay and inhibition of angiogenesis (Yasuda et al, 2001, 2003; Arcasoy et al, 2002).

There have been no reports to date precisely analyzing the angiogenic potential of therapeutic rEpo in an in vivo tumour model. Our laboratory has extensively utilized the rodent dorsal skin flap window chamber model in determining real-time, sequential angiogenic effects of various agents (Li et al, 2000). A limitation of the dorsal skin fold model, however, has been the necessity of growing tumours in an ectopic site. Recently, our laboratory has developed an orthotopic breast window, as described by Shan (Shan et al, 2003). It has been established that tumour microenvironment plays an influential role in the behavior of the tumour itself and that tumours originating from different tissues differ in their phenotypes, biological behaviors, and responses to therapeutic agents (Fidler et al, 1994). When tested in this highly translational in vivo model, rEpo did not demonstrate any proangiogenic activity.

Finally, our studies offer insight into possible clinical effects of rEpo on patients receiving the drug to treat anemia. Two recent clinical trials (Henke et al, 2003; Leyland-Jones, 2003) have suggested that rEpo might negatively impact treatment outcome in cancer patients receiving chemotherapy. Additionally, in vitro evidence suggests that rEpo may induce a cytoprotective phenotype in certain tumour types, which could translate into resistance to certain chemotherapeutics (Belenkov et al, 2004; $\mathrm{McBroom}$ et al, 2005). However, a recent meta-analysis of rEpo used in both patients with anemia and without anemia suggests a strong trend towards a survival advantages in favor of the use of rEpo (Bohlius et al, 2005). Although not combined with chemotherapy or radiation therapy, the present study is the first to provide preclinical in vivo insight into this seemingly confusing clinical situation. In addition, our study suggests that recent clinical results using rEpo in nonanemic patients may not be due to the direct effects of the drug on the tumour, as we did not detect any tumour growth enhancement with rEpo. Our findings warrant further preclinical and clinical investigation of rEpo in order to further clarify the risks of its use as well as optimize its known benefits. 


\section{A}
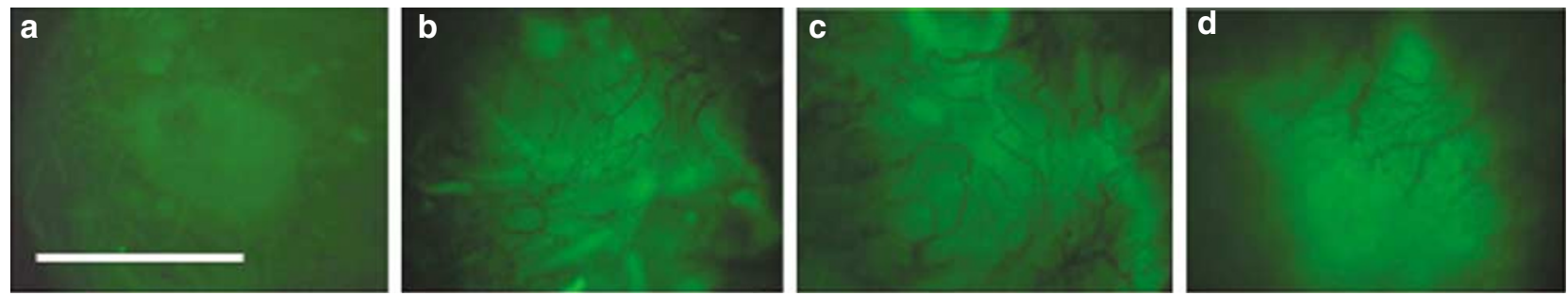

\section{B}
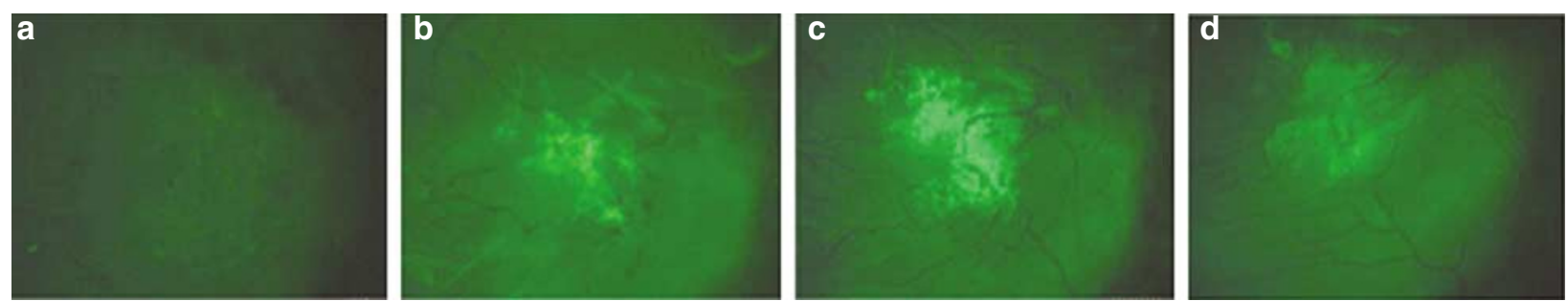

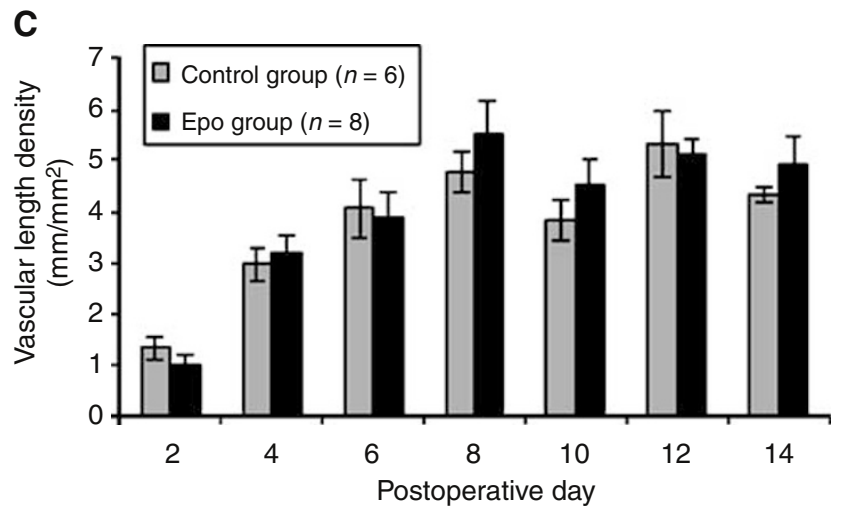

Figure 4 Intravital microscopy of mammary window chamber. Shown are a series of representative images from a rat in the control group (A) and the rEpo-treated group $(\mathbf{B})$ and the effects of $r$ Epo on tumour vascularity $(\mathbf{C})$. Shown images from the mammary window chamber were taken at postoperative day 2 (a), 6 (b), 8 (c), and 14 (d). On postoperative day 2, the tumour size is relatively small, and little angiogenesis has occurred. By postoperative day 6 , the tumour has increased in size and amassed a considerable vasculature, which continues to increase until around postoperative day $12-14$, when the tumour and associated vasculature begin to reach a plateau. Vascular length density (VLD) was calculated for each animal at each time point. At all time points, there was no statistical difference $(P>0.05)$ in the VLD of the treatment and control groups. Values represent the mean of each treatment group \pm standard error of the mean (s.e.m.) at each time point. Scale bar $=300 \mathrm{~m}$.

\section{REFERENCES}

Acs G, Acs P, Beckwith SM, Pitts RL, Clements E, Wong K, Verma A (2001) Erythropoietin and erythropoietin receptor expression in human cancer. Cancer Res 61: $3561-3565$

Anagnostou A, Lee ES, Kessimian N, Levinson R, Steiner M (1990) Erythropoietin has a mitogenic and positive chemotactic effect on endothelial cells. Proc Natl Acad Sci USA 87: 5978-5982

Arcasoy MO, Amin K, Karayal AF, Chou SC, Raleigh JA, Varia MA, Haroon ZA (2002) Functional significance of erythropoietin receptor expression in breast cancer. Lab Invest 82: $911-918$

Bedford JS, Mitchell JB (1974) The effect of hypoxia on the growth and radiation response of mammalian cells in culture. $\mathrm{Br} \mathrm{J}$ Radiol 47: $687-696$

Belenkov AI, Shenouda G, Rizhevskaya E, Cournoyer D, Belzile JP, Souhami L, Devic S, Chow TY (2004) Erythropoietin induces cancer cell resistance to ionizing radiation and to cisplatin. Mol Cancer Ther 3: $1525-1532$
Blackwell KL, Kirkpatrick JP, Snyder SA, Broadwater G, Farrell F, Jolliffe L, Brizel DM, Dewhirst MW (2003) Human recombinant erythropoietin significantly improves tumor oxygenation independent of its effects on hemoglobin. Cancer Res 63: 6162-6165

Bohlius J, Langensiepen S, Schwarzer G, Seidenfeld J, Piper M, Bennett C, Engert A (2005) Recombinant human erythropoietin and overall survival in cancer patients: results of a comprehensive meta-analysis. J Nat Cancer Inst 97: 489-498

Carlini RG, Alonzo EJ, Dominguez J, Blanca I, Weisinger JR, Rothstein M, Bellorin-Font E (1999) Effect of recombinant human erythropoietin on endothelial cell apoptosis. Kidney Int 55: 546-553

Carlini RG, Reyes AA, Rothstein M (1995) Recombinant human erythropoietin stimulates angiogenesis in vitro. Kidney Int 47: 740-745

Dachs GU, Chaplin DJ (1998) Microenvironmental control of gene expression: implications for tumor angiogenesis, progression, and metastasis. Semin Radiat Oncol 8: 208-216 
Fidler IJ, Wilmanns C, Staroselsky A, Radinsky R, Dong Z, Fan D (1994) Modulation of tumor cell response to chemotherapy by the organ environment. Cancer Metastasis Rev 13: 209-222

Graeber TG, Osmanian C, Jacks T, Housman DE, Koch CJ, Lowe SW, Giaccia AJ (1996) Hypoxia-mediated selection of cells with diminished apoptotic potential in solid tumours. Nature 379: 88-91

Henke M, Laszig R, Rube C, Schafer U, Haase KD, Schilcher B, Mose S, Beer KT, Burger U, Dougherty C, Frommhold H (2003) Erythropoietin to treat head and neck cancer patients with anaemia undergoing radiotherapy: randomised, double-blind, placebo-controlled trial. Lancet 362: 1255-1260

Huang Q, Shan S, Braun RD, Lanzen J, Anyrhambatla G, Kong G, Borelli M, Corry P, Dewhirst MW, Li CY (1999) Noninvasive visualization of tumors in rodent dorsal skin window chambers. Nat Biotechnol 17: $1033-1035$

Krantz SB (1991) Erythropoietin. Blood 77: 419-434

Leyland-Jones B (2003) Breast cancer trial with erythropoietin terminated unexpectedly. Lancet Oncol 4: 459-460

Li CY, Shan S, Huang Q, Braun RD, Lanzen J, Hu K, Lin P, Dewhirst MW (2000) Initial stages of tumor cell-induced angiogenesis: evaluation via skin window chambers in rodent models. J Natl Cancer Inst 92: $143-147$

Mancuso P, Burlini A, Pruneri G, Goldhirsch A, Martinelli G, Bertolini F (2001) Resting and activated endothelial cells are increased in the peripheral blood of cancer patients. Blood 97: 3658-3661

McBroom JW, Acs G, Rose GS, Krivak TC, Mohyeldin A, Bell A, Verma A (2005) Erythropoeitin receptor function and expression in epithelial ovarian carcinoma. Gynecol Oncol Epub ahead of print, available online 26 July 2005, in press
Monestiroli S, Mancuso P, Burlini A, Pruneri G, Dell'Agnola C, Gobbi A, Martinelli G, Bertolini F (2001) Kinetics and viability of circulating endothelial cells as surrogate angiogenesis marker in an animal model of human lymphoma. Cancer Res 61: $4341-4344$

Reynolds TY, Rockwell S, Glazer PM (1996) Genetic instability induced by the tumor microenvironment. Cancer Res 56: 5754-5757

Ribatti D, Presta M, Vacca A, Ria R, Giuliani R, Dell'Era P, Nico B, Roncali L, Dammacco F (1999) Human erythropoietin induces a pro-angiogenic phenotype in cultured endothelial cells and stimulates neovascularization in vivo. Blood 93: 2627-2636

Shan S, Sorg B, Dewhirst MW (2003) A novel rodent mammary window of orthotopic breast cancer for intravital microscopy. Microvasc Res 65: $109-117$

Westenfelder C, Baranowski RL (2000) Erythropoietin stimulates proliferation of human renal carcinoma cells. Kidney Int 58: 647-657

Yasuda Y, Fujita Y, Masuda S, Musha T, Ueda K, Tanaka H, Fujita H, Matsuo T, Nagao M, Sasaki R, Nakamura Y (2002) Erythropoietin is involved in growth and angiogenesis in malignant tumours of female reproductive organs. Carcinogenesis 23: $1797-1805$

Yasuda Y, Fujita Y, Matsuo T, Koinuma S, Hara S, Tazaki A, Onozaki M, Hashimoto M, Musha T, Ogawa K, Fujita H, Nakamura Y, Shiozaki H, Utsumi H (2003) Erythropoietin regulates tumour growth of human malignancies. Carcinogenesis 24: $1021-1029$

Yasuda Y, Musha T, Tanaka H, Fujita Y, Fujita H, Utsumi H, Matsuo T, Masuda S, Nagao M, Sasaki R, Nakamura Y (2001) Inhibition of erythropoietin signalling destroys xenografts of ovarian and uterine cancers in nude mice. Br J Cancer 84: 836-843 